\title{
Estudio del concepto de infidelidad en población universitaria
}

\author{
LAURA DOMÍNGUEZ PRADES \\ al135651@uji.es \\ MARÍA GIMENO LOZANO \\ al152152@uji.es \\ RAFAEL BALLESTER-ARNAL \\ rballest@uji.es
}

\section{Resumen}

Introducción: En la actualidad no hay una definición universal acerca del concepto infidelidad. Por ello, este estudio pretende analizar cuál es el concepto de infidelidad que tienen los jóvenes. Método: Los participantes de este estudio son 300 jóvenes universitarios de edades comprendidas entre 18 y 26 años (media $=20,8$, DT $=2,13$ ) equiparados por género. Para la obtención de los datos se ha utilizado un instrumento de elaboración propia que recoge 33 conductas que podrían estar relacionadas con la infidelidad. Cada participante indicó para cada conducta si creía que era una forma de ser infiel y, en el caso de que lo hiciera su pareja, si le parecería que le estaba siendo infiel. Resultados: Se ha obtenido que un $31,5 \%$ de la muestra ha sido infiel, al menos, una vez y un $32,8 \%$ ha sido víctima de una infidelidad. Los resultados muestran que existen diferencias significativas en función del género, siendo las mujeres las que consideran un mayor número de conductas como una manera de ser infiel. Además, se encontraron diferencias significativas en la orientación sexual, obteniendo que las personas con una orientación sexual homosexual eran significativamente más infieles. Discusión: Contrariamente a la percepción de que los hombres cometen más infidelidades que las mujeres, no se han encontrado resultados que sustenten esta afirmación. Así mismo, las conductas en las que se ha encontrado un mayor consenso en cuanto a ser una forma de ser infiel son de carácter sexual.

Palabras clave: infidelidad, concepto, género, prevalencia, tipos.

\section{Abstract}

Introduction: Currently there is no universal definition of infidelity. Therefore, this study aims to analyse young people's concept of infidelity. Method: The participants in this study were 300 university students aged $18-26$ years (average $=20.82, S D=2.134$ ), compared by gender. To obtain data, an instrument was used that the authors developed, which included 33 behaviours possibly related to infidelity. For each behaviour, the participants indicated if they believed it was a way of being unfaithful, and that if their partner 
did this, it would seem (s)he was unfaithful. Results: The obtained results indicated that $31.5 \%$ of the sample had been unfaithful at least once and $32.8 \%$ had been victims of infidelity. They also showed significant differences for gender, as women considered a larger number of behaviours as a way of being unfaithful. Significant differences in sexual orientation were also found as those with a homosexual sexual orientation were significantly more unfaithful than heterosexual and bisexual people. Discussion: No results were found to support the claim that males commit more acts of infidelity than women. The behaviours in which a greater consensus was reached as to the form of being unfaithful were all sexual behaviours.

Keywords: infidelity, concept, gender, prevalence, types.

\section{Introducción}

Diversos autores han intentado elaborar una definición que incluya todas las posibles manifestaciones de la infidelidad. No obstante, actualmente todavía no se ha llegado a consensuar una definición universal. Un ejemplo de definición es la aportada por Blow y Hartnet (2005: 191), que tienen en cuenta los componentes tanto sexuales como emocionales, por lo que definen la infidelidad como «un acto sexual y/o emocional ejercido por una persona dentro de una relación de compromiso, donde se produce un acto de este tipo fuera de la relación primaria y constituye un abuso de confianza y/o violación de normas acordadas (abiertas y encubiertas) por uno o ambos individuos en esa relación en cuanto a la exclusividad romántica/emocional o sexual». Del mismo modo, Weiser, Lalasz, Weigel y Evans (2014) realizaron un estudio con el propósito de conceptualizar la infidelidad, concluyendo que la característica común de todas las definiciones recogidas era el engaño, indicando con ello que la infidelidad se asocia con algún tipo de actitud deshonesta. Además, más de la mitad de los participantes en este estudio definieron la infidelidad como un acto moralmente inapropiado, como algo malo e inmoral y adjudicaron adjetivos como mentiroso, tramposo o poco fiable a las personas que cometen una infidelidad. Por otro lado, desde un punto de vista psicológico, Lewandowsky y Ackerman (2006) hablan de la infidelidad como el resultado de la incapacidad de la relación primaria de llenar ciertas necesidades, por lo que el ser humano se encontraría motivado a buscar a otras personas que satisfagan esas carencias incluso fuera de la relación establecida (Brown, 2002; Medina y cols., 2013).

Otros autores han intentado concretar aquellas conductas o actos que podrían ser considerados como infidelidad. Autores como Allen y Baucom (2006), Brown (2002), Thronton y Nagurney (2011) o Randall y Byers (2003) señalan conductas como tener un desliz (affair), una relación extramarital, poner los cuernos, mantener relaciones sexuales, sexo oral, besarse, acariciarse, tener un vínculo emocional más fuerte que la amistad, relaciones de amistad, relaciones a través de Internet o ver material pornográfico, como aquellas que son percibidas como infidelidad. En lo referente a las diferencias entre géneros, un estudio con muestra universitaria encontró que los hombres se perciben como más suspicaces hacia su pareja, es decir, tienden a pensar más sobre la posibilidad de que les serán infieles en el futuro $\mathrm{y}$, al mismo tiempo, también se perciben como más propensos a ser ellos mismos los que cometan una infidelidad (Goetz y Causey, 2009). Por otro lado, en un estudio realizado con adolescentes, se obtuvo que las mujeres consideran la infidelidad emocional más molesta, al contrario que los hombres, los cuales consideran la infidelidad física más grave (McAnulty y Brineman, 2007). No obstante, existen estudios que han encontrado resultados distintos a los mencionados en función del género (Kruger y cols., 2013). 
A pesar de estos antecedentes, existen pocas investigaciones acerca de la concepción y los significados asociados a la infidelidad, además de existir escasos instrumentos que evalúen dicho concepto. Por esta razón, el presente estudio se centra en averiguar la prevalencia de la infidelidad entre jóvenes universitarios, las conductas que estos consideran como manifestaciones de este fenómeno, la posible existencia de diferencias en la percepción de que ha habido infidelidad en función de que las conductas hayan sido realizadas por uno mismo o por su pareja y las diferencias en todo esto en función del género.

\section{Método}

\section{Participantes}

Se seleccionaron un total de 300 participantes con un rango de edad comprendido entre 18 y 26 años (edad media $=20,82$; DT $=2,13$ ). De esta muestra empleada, 150 participantes (50\%) eran mujeres y 150 (50\%) eran hombres. En cuanto a la nacionalidad de los participantes, se encontraron 7 nacionalidades distintas, siendo la más mentada la española (91\%), seguida de la colombiana (2\%). Por otra parte, también se registraron datos sobre la orientación sexual, con 277 participantes (92,3\%) heterosexuales, 12 (4\%) bisexuales y 9 (3\%) homosexuales. Los dos participantes restantes no especificaron la orientación sexual.

\section{Procedimiento}

En lo que se refiere al procedimiento de recogida de la muestra, se realizaron dos recogidas diferentes. La primera, de forma presencial, durante 4 días por las distintas facultades de la Universitat Jaume I y la segunda opción fue mediante el cuestionario online. En ambos casos, los participantes fueron informados de los objetivos de la investigación y de la confidencialidad con la que se tratarían los datos.

\section{Instrumento de evaluación}

Para llevar a cabo el presente estudio ha sido necesaria la creación de un cuestionario adaptado a los objetivos de la investigación. Para ello, se han tomado como referencia algunos cuestionarios (aunque no validados) que se han centrado en evaluar aspectos similares, como es el caso de The infidelity questionnaire (Thornton y Nagurney, 2011) o The sexual behavior questionnaire (Norona, 2013).

El instrumento final empleado para la recogida de la información consiste en un cuestionario de elaboración propia compuesto por dos partes diferenciadas. Al comienzo, contiene información de tipo demográfica (sexo, edad, nacionalidad, ocupación, orientación sexual, religión). Además, se incluye información sobre relaciones de pareja, pareja en la actualidad o el número de parejas que ha tenido y su duración. En lo que se refiere a la primera parte del cuestionario, incluye 17 ítems relacionados con información como: si alguna vez ha sido infiel, los motivos, tipo de infidelidad, uso del preservativo en esa situación, consecuencias de la infidelidad, si alguna vez le han sido infiel, celos, conductas de control sobre su pareja o la utilización de los servicios de un/a trabajador/a sexual. La segunda parte está compuesta por 33 ítems que hacen referencia a situaciones que el participante debe evaluar como una posible forma de ser infiel, si lo ha hecho alguna vez, si su pareja lo ha hecho alguna vez y, en el caso de que lo hiciera su pareja, 
si le parecería una forma de ser infiel. Para cada una de las conductas el participante tiene que responder de forma dicotómica (sí/no) a cada uno de estos aspectos.

\section{Análisis de datos}

Se han realizado análisis descriptivos de frecuencias, tablas de contingencias y análisis de Chi-cuadrado. Los análisis estadísticos se realizaron mediante el programa estadístico SPS Statistics versión 23.

\section{Resultados}

\section{Prevalencia de la infidelidad realizada y recibida}

Un 52,8 \% de los participantes tiene pareja en la actualidad (un 93,3\% ha tenido pareja alguna vez). Un $68,5 \%$ nunca había sido infiel a sus parejas, un $28,5 \%$ había sido alguna vez infiel, un 2,3\% había sido infiel bastantes veces y un 0,7 \% había sido infiel muchas veces. De las personas que habían sido infieles, el 12,8 \% expresó que fue con alguien de su mismo sexo y un $91,4 \%$ con una persona de distinto sexo. En cuanto a la pregunta « $i T e$ consta que alguna de tus parejas te ha sido infiel?», un 32,8\% afirma que sí.

Por otra parte, en lo que se refiere a la prevalencia de la infidelidad en función de la orientación sexual, se ha encontrado que las personas con una orientación sexual homosexual son significativamente más infieles que las personas con orientaciones heterosexual y bisexual (véase la tabla 1).

Tabla 1

Prevalencia de la infidelidad en función de la orientación sexual

\begin{tabular}{lcccccc}
\hline & Nunca & $\begin{array}{c}\text { Algunas } \\
\text { veces }\end{array}$ & $\begin{array}{c}\text { Bastantes } \\
\text { veces }\end{array}$ & $\begin{array}{c}\text { Muchas } \\
\text { veces }\end{array}$ & $X^{2}$ & $p$ \\
\hline Heterosexual & $68,7 \%$ & $28,7 \%$ & $1,8 \%$ & $0,7 \%$ & 17,606 & .007 \\
Bisexual & $83,3 \%$ & $16,7 \%$ & $0,0 \%$ & $0,0 \%$ & & \\
Homosexual & $44,4 \%$ & $33,3 \%$ & $22,2 \%$ & $0,0 \%$ & & \\
\hline
\end{tabular}

\section{Diferencias en función del género}

En primer lugar, no se han encontrado diferencias significativas en lo que se refiere a la prevalencia de la infidelidad entre hombres y mujeres. En cuanto a las diferencias de género encontradas en la interpretación de que determinadas conductas son una forma de infidelidad, son de tamaño pequeño, siendo las mujeres las que consideran un mayor número de conductas como una manera de ser infiel. A continuación se detallan los resultados más destacados: «12. Masturbarse pensando en otra persona» $\left(x^{2}=5,54 ; p \leq, 019\right)$, «13. Fantasear sexualmente con otra persona» $\left(x^{2}=5,77 ; p \leq, 016\right)$, «18. Dar un pico a otra persona» $\left(x^{2}=5,12 ; p \leq, 024\right)$, «24. Enviar mensajes con contenido sexual» $\left(X^{2}=6,96 ; p \leq, 008\right)$, «25. Mantener una conversación de chat con contenido 
sexual» $\left(x^{2}=15,98 ; p \leq, 000\right)$, «26. Mantener sexo telefónico» $\left(x^{2}=6,25 ; p \leq, 012\right)$ y «28. Realizar conductas sexuales a través de una webcam» $\left(x^{2}=6,25 ; p \leq, 012\right)$ (véase la tabla 2$)$.

Tabla 2

Conductas en las que hay más o menos acuerdo en función de que las haga uno mismo o la pareja y diferencias de género

\begin{tabular}{|c|c|c|c|c|c|c|c|c|c|c|}
\hline \multirow[b]{2}{*}{ Creo que es una forma de ser infiel... } & \multicolumn{5}{|c|}{ Si lo hago yo } & \multicolumn{5}{|c|}{ Si lo hace mi pareja } \\
\hline & $\begin{array}{c}\text { Total } \\
(\mathbf{*})\end{array}$ & $\begin{array}{c}\text { Hombre } \\
(\%)\end{array}$ & $\begin{array}{c}\text { Mujer } \\
(\%)\end{array}$ & $x^{2}$ & $\mathbf{v}$ & $\begin{array}{c}\text { Total } \\
(\%)\end{array}$ & $\begin{array}{c}\text { Hombre } \\
(\%)\end{array}$ & $\begin{array}{c}\text { Mujer } \\
(\%)\end{array}$ & $x^{2}$ & $\mathbf{v}$ \\
\hline $\begin{array}{l}\text {... mantener relaciones sexuales con otra } \\
\text { persona }\end{array}$ & 98,3 & 88,0 & 98,7 & 0,203 & 0,026 & 95,3 & 94,6 & 96,0 & 0,314 & 0,032 \\
\hline ... practicar sexo oral a otra persona & 97,7 & 96,7 & 98,7 & 1,316 & 0,066 & 95,6 & 93,8 & 97,3 & 2,082 & 0,084 \\
\hline ... masturbar a otra persona & 97,7 & 96.7 & 98,7 & 1,316 & 0,066 & 96,3 & 95,3 & 97,3 & 0,848 & 0.053 \\
\hline ... dar un beso largo (beso con lengua) & 94,6 & 83.3 & 96.0 & 1,085 & 0.060 & 88.8 & 87,2 & 90.7 & 0.828 & 0.056 \\
\hline $\begin{array}{l}\text {... realizar conductas sexuales a través de una } \\
\text { webcam }\end{array}$ & 24.3 & 90.7 & 98.0 & $7.545^{\prime \prime}$ & 0.159 & 92.0 & 87.2 & 96.7 & $8.982 " '$ & 0.173 \\
\hline ... magrearse con otra persona & 93.0 & 81.3 & 94,7 & 1,280 & 0.065 & 89.3 & 86,7 & 91.9 & 2,180 & 0.085 \\
\hline ... mantener sexo telefónico & 91,3 & 87.2 & 95,3 & $6.246^{\circ}$ & 0.145 & 88,6 & 84,6 & 92,7 & $4,870^{\prime}$ & 0.128 \\
\hline ... enviar fotos de ti mismo desnudo & 89,7 & 89.3 & 90,0 & 0,036 & 0,011 & 87.1 & 88,0 & 87,3 & 0,031 & 0.010 \\
\hline ... enviar mensajes con contenido sexual & 89,3 & 84.6 & 94,0 & $6.964 " \prime$ & 0,153 & 86,8 & 82,6 & 90,7 & $4,248^{\prime}$ & 0.118 \\
\hline $\begin{array}{l}\text {... mantener una conversación de chat con } \\
\text { contenido sexual }\end{array}$ & 84,3 & 75,8 & 92,7 & $15,977^{\prime \prime \prime}$ & 0,231 & 83,3 & 77,2 & 89,3 & $7,827^{\prime \prime}$ & 0,163 \\
\hline ... estar enamorado de otra persona & 84,2 & 81,2 & 87,2 & 2,046 & 0,083 & 83.3 & 78,7 & 87,9 & $4,595^{\prime}$ & 0.124 \\
\hline ... tener una cita con otra persona & 75,8 & 72.3 & 79,3 & 2,013 & 0,082 & 74,4 & 72,5 & 76,4 & 0,583 & 0.044 \\
\hline ... recibir fotos sexuales de otra persona & 69.0 & 64.2 & 73.8 & 3.225 & 0.104 & 68.2 & 61,2 & 75.2 & $6.638^{\prime}$ & 0.150 \\
\hline ... coquetearitontear con otra persona & 68.1 & 63.1 & 73.2 & 3.477 & 0.108 & 67.2 & 62,7 & 69.8 & 1.700 & 0.075 \\
\hline ... dar un beso (un pico) & 67,0 & 60.8 & 73,2 & $5.117^{\prime}$ & 0.131 & 66.4 & 63,5 & 69.3 & 1,132 & 0.062 \\
\hline $\begin{array}{l}\text {... desear tener relaciones sexuales con otra } \\
\text { persona }\end{array}$ & 54,8 & 54,7 & 55,0 & 0,003 & 0,003 & 54,8 & 53,1 & 56,8 & 0,407 & 0037 \\
\hline ... hacer cualquier cosa a escondidas de tu pareja & 47,5 & 44.0 & 51,0 & 1,472 & 0,070 & 48.0 & 46,0 & 50.0 & 0,478 & 0.040 \\
\hline ... fantasear sexualmente con otra persona & 34.3 & 27.7 & 40.8 & $5.770^{\circ}$ & 0.139 & 35.4 & 27,2 & 43.3 & $8.444 "=$ & 0.168 \\
\hline ... masturbarse pensando en otra persona & 32,8 & 26,4 & 39,2 & $5,538^{\circ}$ & 0,137 & 34,6 & 27,8 & 41,2 & $5,789^{\circ}$ & 0,140 \\
\hline $\begin{array}{l}\text {... hacer un regalo sentimental, sin motivo } \\
\text { aparente, a otra persona }\end{array}$ & 30,8 & 28.7 & 34.8 & 2.378 & 0.089 & 33.4 & 27,3 & 39.8 & $5.051^{\circ}$ & 0.130 \\
\hline ... ir a un dub de stiptease & 29,2 & 28.1 & 30.2 & 0.180 & 0.023 & 27.6 & 24,7 & 30,4 & 1,216 & 0,064 \\
\hline $\begin{array}{l}\text {... propear a alguien a través de las redes } \\
\text { sociales }\end{array}$ & 28,3 & 27,0 & 29,5 & 0,229 & 0,028 & 29,4 & 26,5 & 32,2 & 1,152 & 0,062 \\
\hline ... sentr atracción por otra persona & 26,0 & 27.0 & 25.0 & 0.158 & 0.023 & 28.4 & 27,8 & 25.0 & 0,317 & 0,033 \\
\hline ... chatear con alguien & 10,0 & 7.4 & 127 & 2.312 & 0.088 & 10.4 & 7.4 & 13.3 & 2,783 & 0,097 \\
\hline ... acanciar (no sexualmente) & 8.1 & 9.5 & 6,7 & 0,786 & 0.052 & 11,2 & 11,0 & 11,4 & 0,015 & 0,007 \\
\hline ... bailar en la císcoteca con otra persona & 8.1 & 10.2 & 6.1 & 1.677 & 0.075 & 9.8 & 11.6 & 7.5 & 1.467 & 0.071 \\
\hline ... ver pornografía & 6.4 & 4.0 & 8.7 & 2.755 & 0.096 & 6.7 & 3.4 & 10.0 & $5.217^{\bullet}$ & 0,132 \\
\hline ... masturbarse a uno mismo & 3.0 & 3.4 & 2.7 & 0.115 & 0.020 & 1.7 & 1.4 & 2.0 & 0.197 & 0.028 \\
\hline ... leer novelas eróticas & 2,3 & 2,7 & 2,0 & 0,146 & 0,022 & 2.0 & 1,4 & 2,7 & 0,667 & 0,047 \\
\hline $\begin{array}{l}\text {... darle a «me gustas a una foto de una persona } \\
\text { atractiva }\end{array}$ & 2.0 & 2.0 & 2.0 & 0.000 & 0.000 & 1.7 & 1.3 & 2.3 & 0.197 & 0.028 \\
\hline ... abrazar a otra persona & 2.0 & 2.0 & 2.0 & 0.000 & 0.000 & 2.7 & 2.0 & 3.3 & 0.487 & 0.040 \\
\hline ... mirar a una persona atractiva & 1.7 & 2.7 & 0.7 & 1.815 & 0.080 & 1.7 & 2.1 & 1,3 & 0.232 & 0,028 \\
\hline ... hacerte fotos con otra persona & 0.7 & 1,3 & 0,0 & 2,014 & 0.082 & 1.0 & 0,7 & 1,3 & 0,330 & 0,033 \\
\hline
\end{tabular}

Note, "p<,05: "p p;01: " "p<,001 
Las diferencias de género encontradas en la interpretación de que determinadas conductas llevadas a cabo por la pareja son una forma de infidelidad son de tamaño pequeño a favor de las mujeres, al igual que en el caso anterior. A continuación se detallan los resultados más destacados: «9. Ver pornografía» $\left(x^{2}=5,23 ; p \leq, 022\right)$, «12. Masturbarse pensando en otra persona» $\left(x^{2}=5,79 ; p \leq, 016\right)$, «13. Fantasear sexualmente con otra persona» $\left(x^{2}=8,44 ; p \leq, 004\right)$, «22. Hacer un regalo sentimental a otra persona, sin motivo aparente» $\left(X^{2}=5,05 ; p \leq, 025\right)$, «23. Recibir fotos sexuales de otra persona» $\left(x^{2}=6,64 ; p \leq, 010\right)$, «24. Enviar mensajes con contenido sexual» $\left(x^{2}=4,25 ; p \leq, 039\right)$, «25. Mantener una conversación de chat con contenido sexual» $\left(X^{2}=7,93 ; p \leq, 005\right)$, «26. Mantener sexo telefónico» $\left(X^{2}=4,87 ; p \leq, 027\right)$, «28. Realizar conductas sexuales a través de una webcam» $\left(x^{2}=8,98 ; p \leq, 003\right)$, «31. Estar enamorado de otra persona» $\left(X^{2}=4,60 ; p \leq, 032\right)$ les parece una infidelidad (véase la tabla 2 ).

\section{Discusión y conclusiones}

En la presente investigación, teniendo en cuenta los datos obtenidos, se ha encontrado que un $31,5 \%$ de la muestra de la población universitaria ha sido infiel al menos una vez, respecto a un $68,5 \%$ que no ha cometido nunca una infidelidad. En esta misma línea, no se han obtenido diferencias significativas con respecto al género, al igual que los resultados obtenidos en el estudio de Randall y Byers (2003).

En investigaciones previas en las que se han evaluado diferencias de género en función del tipo de infidelidad (emocional y física/sexual) se ha obtenido que las mujeres llevarían peor una infidelidad de tipo emocional mientras que los hombres la infidelidad física/sexual (McAnulty y Brineman, 2007; Roscoe, Cavanaugh y Kennedy, 1988). En nuestro estudio se ha observado que tanto hombres como mujeres se sienten afectados en mayor medida por una infidelidad de tipo sexual, no existiendo diferencias significativas entre ellos.

Del mismo modo, en referencia a las conductas consideradas como infidelidad, en la presente investigación se ha encontrado que son las mujeres las que valoran un mayor número de conductas como infidelidad respecto a los hombres. Estos resultados van en consonancia a los hallados en el estudio de Kruger y cols. (2013), donde las mujeres también valoraron un mayor número de conductas como infidelidad. En esta misma línea, en el presente estudio, aquellas conductas que más han sido valoradas como infidelidad tanto por hombres como por mujeres han sido las de carácter sexual, al igual que la valoración de las mismas conductas en el caso que las realice la pareja. A pesar de que tanto hombres como mujeres valoran un mayor número de conductas de infidelidad de carácter físico/sexual, son las mujeres las que consideran la infidelidad emocional más grave en comparación con los hombres, resultados que van en consonancia con los encontrados por Roscoe, Cavanaugh y Kennedy (1988).

Por otro lado, los resultados de este estudio muestran diferencias significativas respecto a la orientación sexual, encontrando que las personas con una orientación homosexual se muestran más infieles respecto a las personas con orientación heterosexual y bisexual. Sin embargo, en la muestra, la población de personas homosexuales no es representativa, por lo que deberían hacerse otros estudios que respalden dichos resultados. No obstante, en estudios similares se obtuvo que las personas con una orientación bisexual se sienten más cómodas teniendo relaciones sexuales fuera de la relación de pareja (Simpson, Wilson y Winterheld, 2004), mientras que las personas con orientación heterosexual y homosexual prefieren la cercanía emocional y el compromiso de pareja (Seal, Agostinelli y Hannett, 1994; Simpson y Gangestad, 1991). 
En conclusión, es complicado conceptualizar una definición consensuada acerca de la infidelidad. No obstante, existe bastante consenso en las conductas que se consideran como infidelidad, sobre todo, aquellas de carácter sexual. Así mismo, las diferencias entre hombres y mujeres no son significativas, al contrario de la creencia popular de que los hombres son más infieles.

\section{Referencias bibliográficas}

Allen, E. S. y Baucom, D. H. (2006). Dating, marital, and hypothetical extradyadic involvements: How do they compare? Journal of Sex Research, 43, 307-317.

Blow, A. J. y Hartnett, K. (2005). Infidelity in committed relationships I: a methodological review. Journal of Marital and Family Therapy, 31, 183-216.

Brown, E. M. (2002). Patterns of infidelity and their treatment. (2. a ed.) Portland: Ringgold Inc.

Goetz, A. T. y Causey, K. (2009). Sex differences in perceptions of infidelity: Men often assume the worst. Evolutionary Psychology, 7, 253-263.

Kruger, D., Fisher, M., Edelstein, R., Chopik, W., Fitzgerald, C. y Strout, S. (2013). Was that Cheating ? Perceptions Vary by Sex, Attachment Anxiety, and Behavior. Evolutionary Psychology, 11, 159-171.

Lewandowsky, G. W. y Ackerman, R. A. (2006). Something's missing: Need fulfillment and selfexpansion as predictors of susceptibility to infidelity. The Journal of Social Psychology, 146, 389-403.

McAnulty, R. D. y Brineman, J. M. (2007). Infidelity in dating relationships. Annual Review of Sex Research, 18, 94-114.

Medina, J., Colín, B., Martínez, M., de Oca, Y., Fuentes, N. y Muñoz, M. (2013). Las causas que Ilevan a la infidelidad: Un análisis por sexo. Acta de Investigación Psicológica, 3, 1271-1279.

Norona, J. C. (2013). Adolescents' Definitions of Cheating in Romantic Relationships. Tennessee, Fl: University of Tennessee.

Randall, H. E. y Byers, E. S. (2003). What is sex? Students' definitions of having sex, sexual partner, and unfaithful sexual behavior. The Canadian Journal of Human Sexuality,12, 87-96.

Roscoe, B., Cavanaugh, L. E. y Kennedy, D. R. (1988). Dating infidelity: Behaviors, reasons and consequences. Adolescence, 23, 35-43.

Seal, D. W., Agostinelli, G. y Hannett, C. A. (1994). Extradyadic romantic involvement: Moderating effects of sociosexuality and gender. Sex Roles, 31, 1-22.

Simpson, J. A. y Gangestad, S. W. (1991). Individual differences in sociosexuality: evidence for convergent and discriminant validity. Journal of Personality and Social Psychology, 60, 870-883.

Simpson, J. A., Wilson, C. L. y Winterheld, H. A. (2004). Sociosexuality and romantic relationships. En J. H. Harvey, A. Wenzel y S. Sprecher (eds.), Handbook of sexuality in close relationships. (pp. 87-112). Mahwah, NJ: Erlbaum.

Thornton, V. y Nagurney, A. (2011). What is infidelity? Perceptions based on biological sex and personality. Psychology Research and Behavior Management, 4, 51-58.

Weiser, D. A., Lalasz, C. B., Weigel, D. J. y Evans, W. P. (2014). A prototype analysis of infidelity. Personal Relationships, 21, 655-675. 